\section{Freqüência e variáveis associadas ao aleitamento materno em crianças com até 12 meses de idade no município de Araçatuba, São Paulo, Brazil}

\author{
Frequency and associated variables to \\ breastfeeding among infant up to 12 \\ months of age in Araçatuba, State of São \\ Paulo, Brazil
}

Nemre Adas Saliba 1

Lívia Guimarães Zina 2

Suzely Adas Saliba Moimaz 3

Orlando Saliba 4

1-4 Departamento de Odontologia Infantil e Social. Faculdade de Odontologia. Campus de Araçatuba. Universidade Estadual Paulista "Júlio de Mesquita Filho" - UNESP. Rua José Bonifácio, 1193. Araçatuba, SP, Brasil. CEP: 16.015-050 E-mail:nemre@foa.unesp.br

\begin{abstract}
Objectives: to evaluate the prevalence of breastfeeding among infants receiving care in Araçatuba, in the State of São Paulo, Brazil; to assess associated variables relating to mother and child and the mother's knowledge regarding the relationship between breastfeeding and oral health.

Methods: cross-sectional study. Data was collected during the National Immunization Campaign, in 2005. One hundred mothers of children up to 12 months of age were interviewed. The frequency of breastfeeding was estimated using a survival analysis and statistical analyses were carried out to assess the association between breastfeeding and independent variables.

Results: the prevalence of breastfeeding, at 6 and 12 months was $22.2 \%$ (exclusive) and $65 \%$ (total). The mean duration of exclusive breastfeeding was 3.65 months. The variables associated with weaning were use of a bottle $(\chi 2=9.537 ; p=0.002)$ and of a pacifier $(\chi 2=14.667 ; p=0.001)$. Few mothers (33) demonstrated knowledge of the influence of breastfeeding on their children's oral health, the dentist being more often mentioned as responsible for providing this information.

Conclusions: the prevalence of breastfeeding was satisfactory, although rates for exclusive breastfeeding were low. The use of a bottle and a pacifier were positively associated as determining factors with weaning. The government, media and health professionals must improve effective actions to promote breastfeeding.
\end{abstract}

Key words Breast feeding, Oral health, Child welfare, Risk factors

\section{Resumo}

Objetivos: avaliar a prevalência do aleitamento materno em crianças assistidas em Araçatuba, São Paulo, Brasil; verificar a associação com variáveis materno-infantis e o conhecimento das mães sobre a relação da amamentação com saúde bucal.

Métodos: estudo transversal. Os dados foram coletados durante a Campanha Nacional de Vacinação, em 2005. Foram entrevistadas 100 mães de crianças com até 12 meses de idade. A freqüencia do aleitamento foi estimada por meio da análise de sobrevivencia, e foram realizadas análises estatisticas para verificação da associação entre aleitamento e variáveis independentes.

Resultados: a prevalência do aleitamento aos $6 e$ 12 meses foi de 22,2\% (exclusivo) e 65\% (total). A duração mediana da amamentação exclusiva foi de 3,65 meses. As variáveis associadas ao desmame foram uso de mamadeira $(\chi 2=9,537 ; p=0,002) e$ chupeta $(\chi 2=14,667 ; p=0,001)$. Poucas mães (33) demonstraram saber a influência do aleitamento sobre a saúde bucal de seus filhos, sendo o cirurgiãodentista o profissional mais citado como responsável por essa informação.

Conclusões: a prevalência da amamentação foi satisfatória, porém foram baixas as taxas de aleitamento exclusivo, e como fatores determinantes destacaram-se o uso de mamadeiras e chupetas associado ao desmame. É dever de órgãos governamentais, meios de comunicação e profissionais de saúde compac-tuarem ações efetivas em prol do aleitamento.

Palavras-chave Aleitamento materno, Saúde bucal, Bem-estar da criança, Fatores de risco 


\section{Introdução}

A lactação é uma das maneiras mais eficientes de atender aos aspectos nutricionais, imunológicos, psicológicos e ao desenvolvimento de uma criança no seu primeiro ano de vida.

O leite materno possui características bioquímicas ideais para o crescimento e desenvolvimento da criança, e substâncias que conferem melhor digestibilidade. Estudos mostram que o leite da mãe possui um efeito protetor contra a mortalidade infantil, prevenindo infecções gastrointestinais, dermatite atópica, alergia alimentar, além do efeito contra a obesidade. Ademais, não representa ônus para o orçamento familiar. 1,2

Entre os benefícios da amamentação natural, encontra-se o estímulo a um correto crescimento e desenvolvimento do sistema estomatognático, prevenindo as más oclusões e a instalação de hábitos bucais deletérios. 3-5 Crianças que nunca receberam aleitamento materno podem apresentar freqüência de hábitos respiratórios e de sucção superior àquelas que foram amamentadas no seio da mãe. 6

Em estudo conduzido por Tomita et al.,7 foi relacionada a associação entre idade de desmame e sucção de chupeta. $\mathrm{O}$ hábito mais freqüente foi o da mamadeira $(67,7 \%)$, porém a chupeta foi o único hábito relacionado à ocorrência de más oclusões (mordida aberta e mordida cruzada).

Com base em evidências científicas, 1 a World Health Organization (WHO) e o orgãos governamentais recomendam a prática do aleitamento materno exclusivo por seis meses, e a manutenção da amamentação acrescida de alimentos complementares até os dois anos de vida ou mais.2,8 Segundo a WHO, nem a administração de chás, água e sucos é recomendada antes dos seis meses. ${ }^{2}$

Estudos envolvendo o perfil alimentar de crianças têm demonstrado haver alguns fatores de risco para a prática da amamentação. O padrão de aleitamento materno pode ser influenciado pelo nível educacional, contexto social, tipo de emprego, estado civil, renda, tipo de parto e estado nutricional materno. 7-12 Um melhor atendimento pré-natal e orientações sobre aleitamento materno podem aumentar a duração da amamentação total e exclusiva.

Em países industrializados e em desenvolvimento, apesar do reconhecimento geral das vantagens do leite materno sobre o artificial, as taxas de amamentação ainda são baixas. ${ }^{13-16}$ Lauer et al,17 ao revisarem levantamentos nacionais de 94 países em desenvolvimento, observaram que entre crianças até seis meses de vida ou menos a prevalência do aleitamento exclusivo foi de $39 \%$, e a ausência da amamentação esteve presente em 5,6\% das amostras. Segundo Ramos e Almeida, 18 no Brasil, observa-se um aumento significativo nas taxas de aleitamento materno ao longo desta última década. Se, em 1989, a Pesquisa Nacional de Saúde e Nutrição demonstrou que apenas $49,9 \%$ das crianças até seis meses de vida eram aleitadas, já em 1996, com a Pesquisa Nacional sobre Demografia e Saúde, o quadro mudou, passando para $59,8 \%$. Na pesquisa mais recente realizada pelo Ministério da Saúde, 19 em 1999, abrangendo as capitais brasileiras, o índice de aleitamento ficou em $72 \%$ nessa mesma idade. Esse crescimento, segundo os órgãos governamentais, se deve à intensificação das ações de incentivo à amamentação e de investimentos nessa área.

Apesar das evidências do aumento na duração da mediana da amamentação em algumas regiões do país, outros trabalhos têm demonstrado que o padrão de aleitamento materno ainda está aquém das recomendações internacionais. As taxas de aleitamento materno aos 12 meses de idade podem variar de $20 \%$ a $70 \%$, em diversas regiões brasileiras: $23,1 \% \mathrm{em}$ Pelotas, Rio Grande do Sul, 10 35\% em Ouro Preto, Minas Gerais; 20 37,3\% na região Nordeste; 21 61,6\% em Itapira, São Paulo;22 e 69,2\% em Feira de Santana, Bahia.11

Estudos para diagnóstico rápido sobre amamentação têm sido indicados em campanhas de vacinação pela maior praticidade, baixo custo e confiabilidade. Diante do atual quadro epidemiológico nacional, a descrição do perfil alimentar em regiões diversas do país torna-se imprescindível para a avaliação da prática do aleitamento materno e planejamento de medidas de intervenção nos serviços de saúde.

A presente investigação teve por objetivo avaliar a freqüência e a duração mediana do aleitamento materno em crianças com até 12 meses de idade atendidas em uma Unidade Básica de Saúde no município de Araçatuba, relacionando-as com variáveis sociodemográficas, maternas, de assistência ao parto e de hábitos de sucção não nutritivos, assim como também se objetivou verificar o conhecimento das mães sobre a relação da amamentação com a saúde bucal.

\section{Métodos}

Este estudo transversal, tipo inquérito, foi realizado durante a primeira fase da Campanha Nacional de Vacinação no Município de Araçatuba, em 2005. Localizado na região noroeste do Estado de São Paulo, Brasil, o município possui uma população 
estimada de 179 mil habitantes, 92,5\% residentes em área urbana. ${ }^{23} \mathrm{O}$ número de nascidos vivos no ano de 2004 foi de 2118 crianças, com taxa de mortalidade infantil de 15,11 por 1000 nascidos vivos. $19 \mathrm{O}$ total de crianças menores de um ano vacinadas na primeira fase da campanha foi 2223 crianças, sendo 197 na Unidade Básica de Saúde (UBS) “Alfredo Dantas". A cobertura vacinal obtida para o município foi de 95,1\% (Secretaria Municipal de Saúde de Araçatuba, dados não publicados).

Em um primeiro momento, foi feito o contato com a Secretaria de Saúde do município, com o intuito de obter autorização para a realização da pesquisa, esclarecendo as autoridades responsáveis sobre a natureza e modo de condução da mesma.

Para a coleta dos dados, foi selecionada a UBS "Alfredo Dantas", por apresentar um maior fluxo de atendimento em relação às outras unidades. Fazendo parte da rede pública de saúde, ela atende a população de 21 bairros em uma grande área periférica do município, de baixa condição socioeconômica.

A amostra foi calculada considerando-se uma diferença aceitável de $10 \%$ e taxas de prevalência de amamentação total de $50 \%$, baseadas em estudos brasileiros realizados anteriormente. 10,11,20-22

O tamanho amostral resultou em 96 indivíduos, ficando estabelecida uma amostra de 100, pela facilidade de computações com um número completo.

Dentro da metodologia proposta, optou-se pela entrevista dirigida, utilizando-se para a coleta das informações um formulário estruturado, com 30 questões fechadas, incluindo dados de identificação da mãe e do lactente, características da assistência hospitalar, perfil alimentar da criança, presença de hábitos de sucção, conhecimento sobre a relação da amamentação com a saúde bucal e acompanhamento odontológico. Os dados foram codificados, duplamente conferidos, digitados e processados utilizando o programa Epi-Info versão 3.2.2.

As mães foram abordadas durante a permanência na fila de atendimento, procurando-se saber a idade de seus filhos. Aquelas que se encaixavam no perfil do estudo, ou seja, tinham filhos com até 12 meses de idade, foram convidadas a participar do mesmo, sendo entrevistadas após a vacinação da criança. A entrevista foi anotada e realizada por um examinador previamente calibrado, sendo obtido o termo de consentimento livre esclarecido das participantes da pesquisa. $\mathrm{O}$ atendimento foi realizado das oito horas da manhã até as cinco horas da tarde, quando então a unidade finalizou as suas atividades. Foi realizado um estudo piloto, para validação e adequação do instrumento de coleta de dados, aferição das dificuldades encontradas e capacitação do pesquisador envolvido no projeto, com mães de crianças com até 12 meses de idade cadastradas na Clínica de Gestantes da Faculdade de Odontologia de Araçatuba da Universidade Estadual Paulista "Júlio de Mesquita Filho" (UNESP).

As definições do aleitamento materno utilizadas foram aquelas recomendadas pela $\mathrm{WHO},{ }^{24}$ sendo classificadas em aleitamento exclusivo, predominante, complementar ou artificial (Tabela 1).

Inicialmente, foram descritas as características maternas e infantis, através das estimativas de prevalência calculadas pelo Epi-Info 3.2.2. Foi realizada análise estatística, ou seja, teste do quiquadrado, para investigar associações entre as variáveis independentes (local de residência, idade materna, cor da pele, profissão, estado civil, escolaridade, renda, número de filhos, aleitamento dos outros filhos, mãe aleitada, sexo da criança, peso da criança, idade gestacional, pré-natal, tipo de parto, atendimento no parto, alojamento conjunto, tempo do nascimento à primeira mamada, uso de mamadeira, hábitos de sucção, acompanhamento odontológico) e o aleitamento materno exclusivo em crianças com até seis meses, bem como o aleitamento materno em crianças com até 12 meses.

A análise da freqüência e duração mediana da amamentação foi calculada através da técnica denominada análise de sobrevivência, ou tábua de vida. 25 Essa técnica permite a estimativa da proporção de crianças amamentadas em diferentes idades, contornada a principal dificuldade neste tipo de estudo, as crianças que ainda estavam sendo amamentadas durante a coleta dos dados. $\mathrm{Na}$ construção da tábua de vida, o abandono total da prática da amamentação e a introdução de outros alimentos, líquidos ou sólidos, atuaram como eventos terminais das tábuas relativas à amamentação e à amamentação exclusiva, respectivamente. Todas as crianças são consideradas como se tivessem mamado pelo menos no primeiro dia. As crianças ainda amamentadas no momento da entrevista foram consideradas censuradas para cada uma das respectivas tábuas. Para as diferentes práticas do aleitamento materno (amamentação e amamentação exclusiva), foi calculada a duração da mediana, que é a idade em que metade das crianças já foram desmamadas.

Esse estudo se enquadra na modalidade de pesquisa de risco mínimo, de acordo com a resolução 196/96 do Conselho Nacional de Saúde, relativa à pesquisa em seres humanos, e teve a aprovação do Comitê de Ética e Pesquisa em Seres Humanos da Faculdade de Odontologia de Araçatuba da UNESP (Proc. FOA 2005-01398). 
Tabela 1

\begin{tabular}{llll}
\hline Critérios para classificação do aleitamento materno. & & \\
\hline Classificação & $\begin{array}{l}\text { Obrigatoriamente, a } \\
\text { criança deve receber }\end{array}$ & $\begin{array}{l}\text { Permite que a criança } \\
\text { receba }\end{array}$ & Não é permitido receber \\
\hline Aleitamento exclusivo & Leite materno & $\begin{array}{l}\text { Vitaminas, sais minerais (na } \\
\text { forma de comprimidos e } \\
\text { xaropes) e medicamentos }\end{array}$ & $\begin{array}{l}\text { Nenhum tipo de suplementos } \\
\text { (líquidos, pastoso ou sólido) }\end{array}$ \\
Aleitamento predominante & $\begin{array}{l}\text { Leite materno como } \\
\text { fonte principal de }\end{array}$ & $\begin{array}{l}\text { Líquidos (água, chá, suco) } \\
\text { nutrição }\end{array}$ & Nenhum tipo de suplemento \\
Aleitamento complementar & $\begin{array}{l}\text { Leite materno a } \\
\text { alimentos pastosos e } \\
\text { sólidos }\end{array}$ & $\begin{array}{l}\text { Qualquer tipo de alimento } \\
\text { ou líquido }\end{array}$ & \\
Leite materno & & & \\
Aleitamento (total) & Alimentos líquidos e & & \\
sólidos & & & \\
\hline
\end{tabular}

Fonte: WHO. Indicators for assessing breastfeeding practices. 1991.24

\section{Resultados}

As Tabelas 2 e 3 apresentam as características da população de estudo. A idade média das mães foi de 26 anos, sendo $29 \%$ adolescentes. A maioria das mães (89\%) vivia junto com seus parceiros, em domicílios localizados na zona urbana (97\%), tendo uma renda familiar média de 2,8 salários mínimos. Apenas oito mulheres haviam iniciado o ensino superior, tendo sido observado que $60 \%$ das mães não trabalhavam. Encontrou-se a proporção de 54\% de primigestas. A idade média dos lactentes foi de 5,8 meses, $65 \%$ deles na faixa etária de zero a seis meses. Houve um equilíbrio na amostra entre meninos (52\%) e meninas (48\%). Apenas 12 crianças nasceram com menos de 37 semanas e peso inferior a $2500 \mathrm{~g}$ (7), consideradas prematuras. Das mães, 99 fizeram o pré-natal, e entre essas, apenas uma recebeu menos de seis consultas. Destacaramse os partos cesarianos (63\%) e o atendimento pelo Sistema Único de Saúde (SUS), correspondendo a $61 \%$ dos partos. O alojamento conjunto foi uma prática prevalente entre as mães entrevistadas.

Entre as multíparas, $87 \%$ haviam amamentado naturalmente seus outros filhos. Setenta e seis mulheres recordaram terem sido elas próprias amamentadas por suas mães.

No momento da coleta dos dados, 20 crianças estavam sob aleitamento materno exclusivo, $21 \mathrm{sob}$ aleitamento materno predominante, 34 , aleitamento materno complementar (34) e 25 desmamadas. A Figura 1 retrata a curva de duração da amamentação exclusiva e total (soma das prevalências do aleitamento exclusivo, predominante e complementar) nas crianças avaliadas. Observa-se a queda acentuada na prática do aleitamento exclusivo durante os primeiros seis meses. No intervalo de 0-30 dias de vida, 75,5\% dos lactentes estavam sendo alimentados exclusivamente com leite materno, caindo essa proporção para $45,1 \%$ no quarto mês, sendo que aos seis meses apenas $22,2 \%$ estavam sob esse regime. A duração mediana da amamentação exclusiva foi de 3,65 meses. Quanto à amamentação total, houve um declínio menos acentuado durante o período relatado; no primeiro mês, a prevalência foi de $92,9 \%, 79,9 \%$ no quarto mês, $67,6 \%$ no sexto mês, e para aqueles maiores de sete meses, a prevalência foi de $65 \%$, mantendo-se estável até os 12 meses. Como a mediana da amamentação é a idade em que metade das crianças já foram desmamadas e para o aleitamento total não houve um valor abaixo de $50 \%$, não foi possível obter esse número.

O tempo decorrido entre o nascimento e a primeira mamada tem sido relatado como um fator chave para o início da amamentação. Esse tempo foi menor que cinco horas em $68 \%$ dos casos obser- 
Características das mães incluídas no estudo ( $n=100)$. Araçatuba, São Paulo, 2005.

\begin{tabular}{|c|c|c|}
\hline Características & $\mathbf{N}$ & $\%$ \\
\hline \multicolumn{3}{|l|}{ Demográficas } \\
\hline \multicolumn{3}{|l|}{ Localização do domicílio } \\
\hline Área urbana & 97 & 97,0 \\
\hline Área rural & 3 & 3,0 \\
\hline \multicolumn{3}{|l|}{ Renda familiar (SM)* } \\
\hline $0-2$ & 48 & 48,0 \\
\hline $3-4$ & 34 & 34,0 \\
\hline $5-7$ & 11 & 11,0 \\
\hline Mais de 7 & 7 & 7,0 \\
\hline \multicolumn{3}{|l|}{ Maternas } \\
\hline \multicolumn{3}{|l|}{ Idade (anos) } \\
\hline $15-20$ & 29 & 29,0 \\
\hline $21-30$ & 46 & 46,0 \\
\hline $31-40$ & 22 & 22,0 \\
\hline Mais de 40 & 3 & 3,0 \\
\hline \multicolumn{3}{|l|}{ Cor** $(n=95)$} \\
\hline Parda & 40 & 42,1 \\
\hline Branca & 37 & 38,9 \\
\hline Negra & 15 & 15,8 \\
\hline Amarela & 3 & 3,2 \\
\hline \multicolumn{3}{|l|}{ Atual situação conjugal } \\
\hline Casada / amasiada & 89 & 89,0 \\
\hline Divorciada & 3 & 3,0 \\
\hline Solteira & 8 & 8,0 \\
\hline \multicolumn{3}{|l|}{ Situação empregatícia } \\
\hline Não trabalha & 60 & 60,0 \\
\hline Empregada & 29 & 29,0 \\
\hline Autônoma & 9 & 9,0 \\
\hline Estudante & 2 & 2,0 \\
\hline \multicolumn{3}{|l|}{ Escolaridade materna } \\
\hline Analfabeta & 2 & 2,0 \\
\hline Ensino fundamental & 26 & 26,0 \\
\hline Ensino médio & 64 & 64,0 \\
\hline Ensino superior & 8 & 8,0 \\
\hline \multicolumn{3}{|c|}{ Número de filhos nascidos e vivos } \\
\hline 1 & 54 & 54,0 \\
\hline 2 & 33 & 33,0 \\
\hline 3 & 8 & 8,0 \\
\hline 4 ou mais & 5 & 5,0 \\
\hline
\end{tabular}

SM = salário mínimo: valores vigentes na época da coleta $(\mathrm{R} \$ 300,00) ; * *$ Número total de resposta inferior a 100.

vados nesse estudo, $25 \%$ ficando entre 15 a 30 minutos.

O perfil alimentar do grupo foi bastante diverso. A Figura 2 mostra o consumo dos principais alimentos utilizados pelas mães na dieta das crianças com até 6 e 12 meses de idade. A introdução de outros alimentos diferentes do leite materno caracteriza o processo de desmame. Observou-se a preco- cidade na oferta de alimentos líquidos, pastosos e sólidos na amostra estudada, com especial atenção ao consumo de refrigerantes. Entre os lactentes com até 180 dias $(n=67), 47,7 \%$ tomavam água, 41,5\% chá, 36,9\% suco, 35,4\% leite de saquinho/caixinha, $10,8 \%$ fórmula infantil, $4,6 \%$ refrigerante; $32,3 \%$ recebiam alimentos pastosos e 10,8\% alimentos sólidos. A oferta desses alimentos foi maior nos 
Tabela 3

Características das crianças incluídas no estudo $(\mathrm{N}=100)$ e de assistência ao parto. Araçatuba, São Paulo, 2005.

\begin{tabular}{|c|c|c|}
\hline Características & $\mathbf{N}$ & $\%$ \\
\hline \multicolumn{3}{|l|}{ Infantil } \\
\hline $1-3$ & 37 & 37,0 \\
\hline $4-6$ & 28 & 28,0 \\
\hline $10-12$ & 19 & 19,0 \\
\hline \multicolumn{3}{|l|}{ Gênero } \\
\hline Feminino & 48 & 48,0 \\
\hline Masculino & 52 & 52,0 \\
\hline \multicolumn{3}{|l|}{ Peso ao nascer (gramas)* $(n=99)$} \\
\hline $3.501-.4 .000$ & 25 & 25,3 \\
\hline \multicolumn{3}{|l|}{ Idade gestacional } \\
\hline Menos 37 semanas & 12 & 12,0 \\
\hline 37 semanas ou mais & 88 & 88,0 \\
\hline \multicolumn{3}{|l|}{ Assistência ao parto } \\
\hline \multicolumn{3}{|l|}{ Tipo de parto } \\
\hline Cesariano & 63 & 63,0 \\
\hline Normal & 37 & 37,0 \\
\hline Não & 15 & 15,2 \\
\hline
\end{tabular}

* Número total de respostas inferior a 100

Figura 1

Comparação entre as curvas de duração da amamentação total e exclusiva em crianças com até 12 meses de idade. Araçatuba, São Paulo, 2005.

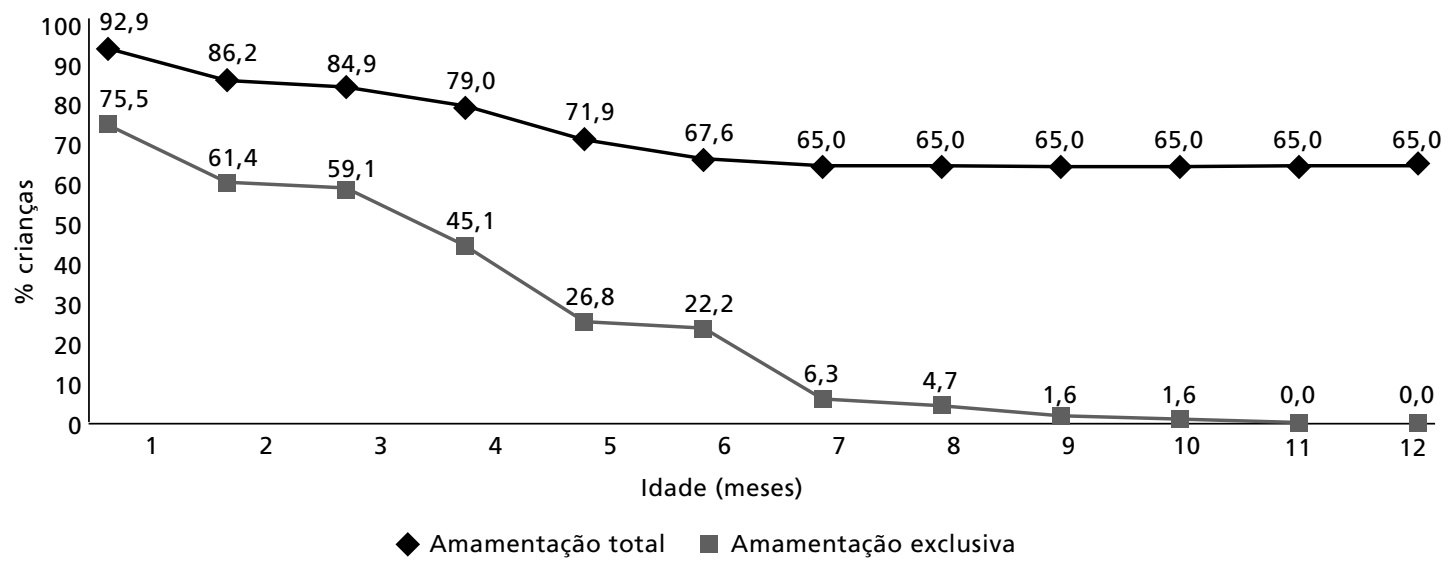




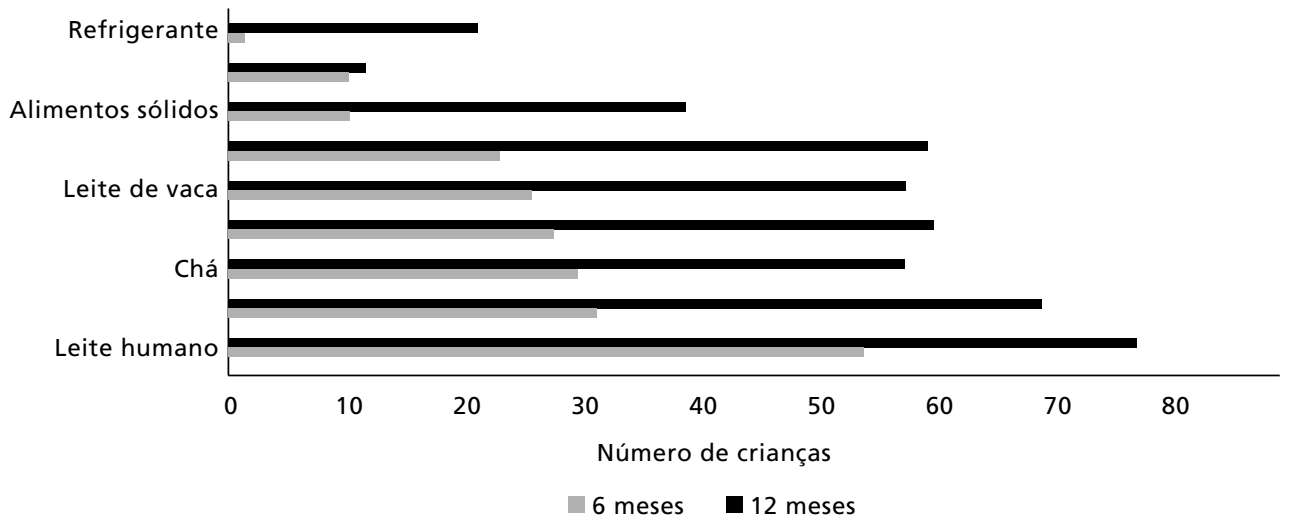

meses subseqüentes. Avaliando o grupo todo, ou seja, crianças com até 12 meses $(n=100), 66 \%$ tomavam água, $51 \%$ chá, $57 \%$ suco, $50 \%$ leite de saquinho/caixinha, $8 \%$ fórmula infantil, $20 \%$ refrigerante; $56 \%$ recebiam alimentos pastosos e $36 \%$ alimentos sólidos. Em relação ao consumo de refrigerantes, foram observadas as seguintes idades e o número correspondentes de crianças: três meses (1), cinco meses (2), sete meses (1), oito meses (5), nove meses (2), 10 meses (1), 11 meses (6), 12 meses (2), totalizando $20 \%$ da amostra.

A prevalência dos hábitos de sucção não nutritivos é apresentada na Figura 3. Estão incluídos nessa categoria os bicos artificiais, como a mamadeira (71\%) e a chupeta (42\%), a sucção digital (8\%), chupeta e sucção digital concomitantemente (3\%).

Observou-se, na análise estatística, associação significativa entre o aleitamento materno exclusivo até seis meses e o uso de mamadeira $\left(\aleph^{2}=35,843\right.$; $)$ $(p<0,001)$ (Tabela 4). Para o aleitamento materno total até 12 meses, estiveram associados o uso de mamadeira $\left(\aleph^{2}=9,537\right) ;(p=0,002)$ (Tabela 5), chupeta $\left(\aleph^{2}=14,667 ; p=0,001\right)$ (Tabela 6) e a ausência de hábitos de sucção $\left(\aleph^{2}=12,943\right)$; $(p=0,001)$ (Tabela 7).

Não foram encontradas associações estatisticamente significativas entre o aleitamento materno, fosse exclusivo ou não, e variáveis sócio-demográficas, maternas e de assistência ao parto $(p>0,05)$.

Procurou-se averiguar o conhecimento das mães sobre os efeitos da amamentação no desenvolvimento das estruturas da face e, conseqüentemente na saúde bucal. O conhecimento dessa relação não é de domínio popular e menos da metade das mães $(n=33)$ demonstraram saber a influência que o aleitamento
Figura 2

Hábitos de sucção não nutritivos de crianças com até 12 meses de idade relatados por suas mães. Araçatuba, São Paulo, 2005.

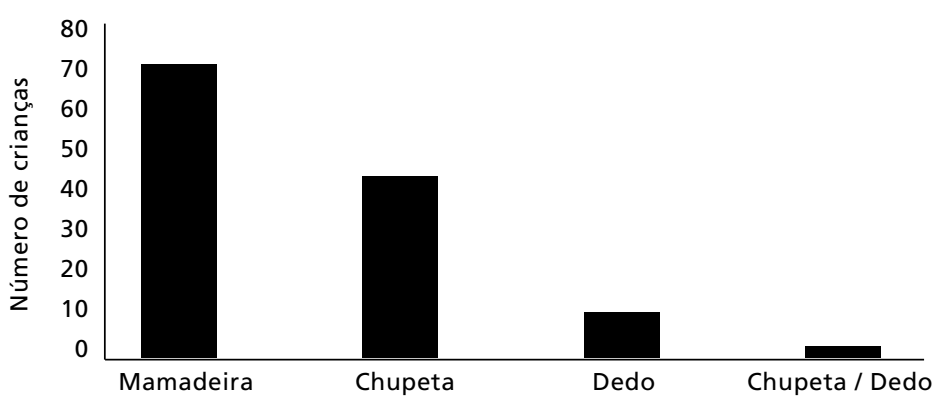

pode exercer sobre a saúde bucal de seus filhos.

Alguns atores sociais estiveram envolvidos na divulgação desse conhecimento. Foram citados pelas mães, como responsáveis por fornecer informação sobre a relação da amamentação com a saúde bucal dos bebês, os seguintes sujeitos: cirurgião-dentista $(33,3 \%)$, médico $(24,2 \%)$, enfermeira $(9,1 \%)$, familiares e amigos $(9,1 \%)$. Meios de comunicação, como cartazes e panfletos $(9,1 \%)$, televisão e rádio $(12,1 \%)$ também estiveram presentes nos discursos.

Os profissionais de saúde também foram citados pelas mães como facilitadores do aleitamento ao lhes oferecerem apoio enquanto amamentavam seus filhos.

Apenas 29 mães receberam tratamento odontológico durante a gestação e entre as 71 que não receberam, apenas 42 relataram sentir falta de acompanhamento por um odontólogo. 
Tabela 4

Distribuição das crianças em relação ao uso de mamadeira e prevalência de aleitamento materno exclusivo até seis meses de idade. Araçatuba, São Paulo, 2005.

\begin{tabular}{lccc}
\hline Mamadeira & Aleitamento exclusivo & Não exclusivo & Total \\
\hline Presente & $18(30 \%)$ & $6(10 \%)$ & $24(39 \%)$ \\
Ausente & $0(0 \%)$ & $37(61 \%)$ & $37(61 \%)$ \\
Total & $18(30 \%)$ & $43(70 \%)$ & $61(100 \%)$ \\
\hline
\end{tabular}

Teste do qui-quadrado: $\kappa 2=35,843 ; p<0,001$

\section{Tabela 5}

Distribuição das crianças em relação ao uso de mamadeira e prevalência de aleitamento materno total até 12 meses de idade. Araçatuba, São Paulo, 2005.

\begin{tabular}{lccc}
\hline Mamadeira & Aleitamento exclusivo & Não exclusivo & Total \\
\hline Presente & $0(0 \%)$ & $25(26 \%)$ & $25(26 \%)$ \\
Ausente & $24(25 \%)$ & $47(49 \%)$ & $71(741 \%)$ \\
Total & $24(25 \%)$ & $72(75 \%)$ & $96(100 \%)$ \\
\hline
\end{tabular}

Teste do qui-quadrado: $\kappa 2=9,537 ; p=0,002$

Tabela 6

Distribuição das crianças em relação ao uso de chupeta e prevalência de aleitamento materno total até 12 meses de idade. Araçatuba, São Paulo, 2005.

\begin{tabular}{lccc}
\hline Mamadeira & Aleitamento exclusivo & Não exclusivo & Total \\
\hline Presente & $20(20 \%)$ & $25(25 \%)$ & $45(45 \%)$ \\
Ausente & $5(5 \%)$ & $50(50 \%)$ & $55(55 \%)$ \\
Total & $25(25 \%)$ & $75(75 \%)$ & $100(100 \%)$ \\
\hline
\end{tabular}

Teste do qui-quadrado: $\kappa 2=14,667 ; p=0,001$

\section{Tabela 7}

Distribuição das crianças incluídas em relação à presença de hábitos de sucção não nutritivos e prevalência de aleitamento materno total até 12 meses de idade. Araçatuba, São Paulo, 2005.

\begin{tabular}{lccc}
\hline Mamadeira & Aleitamento exclusivo & Não exclusivo & Total \\
\hline Ausente & $3(3 \%)$ & $42(42 \%)$ & $45(45 \%)$ \\
Presente & $22(22 \%)$ & $33(33 \%)$ & $55(55 \%)$ \\
Total & $25(25 \%)$ & $75(75 \%)$ & $100(100 \%)$ \\
\hline
\end{tabular}

Teste do qui-quadrado: $\kappa 2=12,943 ; p=0,001$

\section{Discussão}

A prevalência do aleitamento materno, principalmente exclusivo, tem apresentado uma queda acentuada ao longo do primeiro ano de vida das crianças brasileiras, embora essas taxas venham aumentando nos últimos anos.11,22,26

Os valores obtidos neste estudo $(22 \%$ em regime exclusivo aos seis meses e $65 \%$ amamentadas aos 12 meses) foram superiores àqueles encontrados no estudo nacional de 199919 (9,7\% sob aleitamento exclusivo aos seis meses e $44,2 \%$ das crianças amamentadas aos 12 meses). Também estiveram acima dos valores observados em municípios da região Sudeste 20 e Nordeste 21 do Brasil. Neste último trabalho foi observada uma alta taxa de desmame na população de estudo, já que aos 12 meses, apenas $37,3 \%$ das crianças continuavam mamando.

A comparação dos resultados dessas pesquisas demonstra que, apesar da multiplicidade geográfica, as deficiências são semelhantes e devem ser compartilhadas para que ações abrangentes sejam instituídas como medidas de combate ao desmame precoce no país.

Um inquérito sobre as práticas de aleitamento conduzido pela Secretaria de Saúde do Estado e a Faculdade de Saúde Pública, em 1998, realizado em 84 municípios do Estado de São Paulo, encontrou como fatores associados à interrupção do aleitamento materno a ausência do Programa Hospital Amigo da Criança, primiparidade e trabalho materno. Quanto ao aleitamento materno exclusivo em menores de quatro meses, foram identificadas também a escolaridade materna e a maternidade precoce. A análise dos fatores estudados mostrou que o trabalho informal e o desemprego influenciam no desmame precoce. 26

Quanto aos hábitos de sucção, foram observadas relações estatisticamente significantes entre o uso de mamadeira/chupeta e aleitamento. As crianças que usavam mamadeira estiveram mais propensas à interrupção do aleitamento ou à introdução de novos alimentos. Também o uso de chupetas esteve associado ao desmame. A ausência de hábitos de sucção foi considerada um fator protetor da amamentação.

Mamadeiras e chupetas já haviam sido associadas ao desmame precoce por Victora et al.27 Segundo esses autores, esses utensílios são utilizados, em algumas situações, como instrumentos para desmamar a criança, substituir o "peito" da mãe, diminuindo o estímulo ao mamilo e, portanto, reduzindo a produção láctea.

Sabe-se que o aleitamento materno auxilia no 
correto desenvolvimento das estruturas orofaciais, 6 podendo ser considerado um fator preventivo para a instalação de hábitos de sucção. ${ }^{28}$

Tem sido demonstrado que o uso de chupeta logo após o nascimento, durante o período em que a criança está aprendendo a sugar o leite do seio da mãe, pode interferir com o desenvolvimento de uma sucção adequada e contribuir para que a criança confunda o seio com a chupeta, trocando o primeiro por esta última. ${ }^{29}$ Os bicos artificiais podem alterar o desenvolvimento normal da arcada dentária, promovendo maiores índices de alterações oclusais. A fonação pode futuramente também ser comprometida devido às inadequações dos órgãos fonoarticulatórios (sucção com pressão negativa). ${ }^{5}$ Para Planas, 3 a amamentação materna representa, em uma instância maior, a "terapêutica precoce de reabilitação neuroclusal".

A introdução precoce de outros alimentos na dieta da criança em idade de aleitamento, principalmente a suplementação de água e chás, não é problema exclusivo do município de Araçatuba. Situação semelhante é relatada em outros estudos. Em Ouro Preto, Minas Gerais, em 76,4\% das crianças que tiveram suplementação de chás, isso ocorreu antes de completarem 1 mês de vida. 20 Entre os lactentes menores de 90 dias de vida no município de Itapira, 23,6\% tomavam água, e 24,8\% tomavam chá, sendo a oferta de água crescente nos meses subseqüentes. 22

Sobre a suplementação alimentar, além da administração precoce de alimentos em um período no qual somente a oferta de leite materno é suficiente para o adequado desenvolvimento infantil, destacou-se, no presente trabalho, a introdução dos refrigerantes na dieta das crianças.

Muito se tem falado sobre os benefícios do aleitamento, mas pouco destaque é dado ao desenvolvimento do sistema estomatognático, ficando este conhecimento circunscrito à Academia.2,5,30 A divulgação desse tipo de informação pode contribuir como um motivo a mais para as mães optarem pela amamentação, já que a conscientização é mola propulsora para a mudança de atitude do ser humano.

A educação pré-natal é uma importante ferra-

\section{Referências}

1. WHO (World Health Organization). The optimal duration of exclusive breastfeeding: a systematic review. Geneva; 2001 . menta de auxílio à promoção do aleitamento materno. ${ }^{2}$ Estratégias de intervenção, como discussões em grupo abordando os mitos, inibições e demonstrações práticas, além de palestras sobre as vantagens da amamentação, com a participação de pessoas do convívio social da mulher, tais como o pai, avós e amigas íntimas, são métodos úteis e eficazes que devem ser implementados. Outras alternativas viáveis são a formação de grupos de apoio entre as mães, visitas domiciliares realizadas por agentes comunitários e a educação comunitária durante a gestação.

Apesar dos esforços que vêm sendo realizados a favor da amamentação, em todas as áreas de abrangência - local, estadual e nacional -, o aleitamento materno, em Araçatuba, assim como em diversas regiões brasileiras, ainda está aquém dos índices recomendados pela Organização Mundial da Saúde.2,8 As atividades de promoção de saúde devem ser direcionadas a grupos de risco, sendo enfatizados os prejuízos do uso da chupeta e mamadeira ao desmame precoce e suas conseqüências. Nesse sentido, tanto os órgãos governamentais e institucionais, quanto os meios de comunicação e os profissionais de saúde, de modo individual e coletivo, devem compactuar ações efetivas em prol do aleitamento.

Embora a população desse estudo não seja uma amostra representativa do município, os resultados encontrados possibilitam um diagnóstico rápido das práticas de amamentação infantil nos bairros atendidos pela Unidade Básica de Saúde, contribuindo para subsidiar o planejamento de intervenções apropriadas voltadas à promoção do aleitamento materno entre os usuários dos serviços de saúde, visto que existem semelhanças entre os aspectos mais relevantes desse estudo e outros similares realizados em outros municípios e regiões com características sócio-demográficas semelhantes.

\section{Agradecimentos}

Agradecemos à Coordenação de Aperfeiçoamento de Pessoal de Ensino Superior (CAPES) pelo suporte financeiro destinado à realização desta pesquisa.

2. OMS (Organização Mundial da Saúde). Evidências científicas dos dez passos para o sucesso no aleitamento materno. Brasília, DF; 2001. 
3. Planas P. Reabilitação neuroclusal. 2. ed. Rio de Janeiro: Medsi; 1997.

4. Bittencourt LP, Modesto A, Bastos EPS. Influência do aleitamento sobre a freqüência dos hábitos de sucção. Rev Bras Odontol. 2001; 58: 191-3.

5. Carvalho GD. Amamentação e o sistema estomatognático. In: Carvalho MR, Tamez RN. Amamentação: bases científicas para a prática profissional. Rio de Janeiro: Guanabara Koogan; 2002. p. 37-49.

6. Charchut SW, Allred EN, Needleman HL. The effects of infant feeding patterns on the occlusion of the primary dentition. J Dent Child (Chic ) 2003; 70:197-203.

7. Tomita LM, Carrascoza KC, Possobon RF, Ambrosano GMB, Moraes ABA. Relação entre o tempo de aleitamento materno, introdução de hábitos orais e ocorrência de más oclusões. Rev Fac Odontol Univ Passo Fundo. 2004; 9: 101-4.

8. WHO (World Health Organization). Global strategy for infant and young child feeding. Geneva; 2003.

9. Kummer SC, Giugliani ERJ, Susin LO, Folletto JL, Lermen NR, Wu VYJ, Santos L, Caetano MB. Evolução do padrão de aleitamento materno. Rev Saúde Pública. 2000; 34: 1438.

10. Horta BL, Olinto MTA, Victora CG, Barros FC, Guimarães PRV. Amamentação e padrões alimentares em crianças de duas coortes de base populacional no sul do Brasil: tendências e diferenciais. Cad Saúde Pública. 1996; 12 (Supl 1): 43-8.

11. Vieira GO, Almeida JAG, Silva LR, Cabral VA, Santana Netto PV. Fatores associados ao aleitamento materno e desmame em Feira de Santana, Bahia. Rev Bras Saúde Matern Infant. 2004; 4: 143-50.

12. Dubois L, Girard M. Social determinants of initiation, duration and exclusivity of breastfeeding at the population level: the results of the longitudinal study of child development in Quebec (ELDEQ 1998-2002). Can J Public Health. 2003; 94: 300-305.

13. Taylor JS, Risica PM, Geller L, Kirtania U, Cabral HJ. Duration of breastfeeding among first-time mothers in the United States: results of a national survey. Acta Paediatr. 2006; 95: 980-4.

14. Xu F, Binns C, Zheng S, Wang Y, Zhao Y, Lee A. Determinants of exclusive breastfeeding duration in Xinjiang, PR China. Asia Pac J Clin Nutr. 2007; 16: 31621.

15. Espinoza H. The relationship between family structure and exclusive breastfeeding prevalence in Nicaragua. Salud Pública Méx. 2002; 44: 499-507.

16. Schluter PJ, Carter S, Percival T. Exclusive and any breastfeeding rates of Pacific infants in Auckland: data from the Pacific Islands families first two years of life study. Public Health Nutr. 2006; 9: 692-699.

Recebido em 25 de janeiro de 2007

Versão final apresentada em: 28 de julho de 2008

Aprovada em 4 setembro e 2008
17. Lauer JA, Betran AP, Victora CG, de Onis M, Barros AJ. Breastfeeding patterns and exposure to suboptimal breastfeeding among children in developing countries: review and analysis of nationally representative surveys. BMC Med. 2004; 2: 1-29.

18. Ramos CV, Almeida JAG. Alegações maternas para o desmame: estudo qualitativo. J Pediatr (Rio J). 2003; 79: $385-90$.

19. Brasil. Ministério da Saúde. Secretaria de Políticas de Saúde. Estudos amostrais. Indicadores Dados Básicos-2004: indicadores de morbidade e fatores de risco. [acesso em: 1 nov. 2005]. Disponível em: http:// www.tabnet.datasus.gov.br/cgi/idb2004/ d19.htm

20. Passos MC, Lamounier JA, Silva CAM, Freitas SN, Baudson MFR. Práticas de amamentação no município de Ouro Preto, MG, Brasil. Rev Saúde Pública. 2000; 34: 61722.

21. Lima TM, Osório MM. Perfil e fatores associados ao aleitamento materno em crianças menores de 25 meses da Região Nordeste do Brasil. Rev Bras Saúde Matern Infant. 2003; 3 : 305-14.

22. Audi CAF, Corrêa AMS, Latorre MRDO. Alimentos complementares e fatores associados ao aleitamento materno e ao aleitamento materno exclusivo em lactentes até 12 meses de vida em Itapira, São Paulo, 1999. Rev Bras Saúde Matern Infant. 2003; 3: 85-93.

23. IBGE (Instituto Brasileiro de Geografia e Estatística). Cidades. [acesso em 1 nov. 2005]. Disponível em: http://www.ibge.gov.br/cidadesat/default.php

24. WHO (World Health Organization). Indicators for assessing breastfeeding practices. Geneva; 1991.

25. Barros FC, Victora CG. Epidemiologia da saúde infantil: um manual para diagnósticos comunitários. 3. ed. São Paulo: Hucitec, Unicef; 1998.

26. Venancio SI, Escuder MML, Kitoko P, Rea MF, Monteiro CA. Freqüência e determinantes do aleitamento materno em municípios do Estado de São Paulo. Rev Saúde Pública. 2002; 36: 313-8.

27. Victora CG, Behague DP, Barros FC, Olinto MT, Weiderpass E. Pacifiers use and short breastfeeding duration: cause, consequence or coincidence? Pediatrics. 1997; 99: 445-53.

28. Howard CR, Howard FM, Lanphear B, Eberly S, deBlieck EA, Oakes D, Lawrence RA. Randomized clinical trial of pacifier use and bottle-feeding or cupfeeding and their effect on breastfeeding. Pediatrics 2003; 111:511-8.

29. Neifert M, Lawrence R, Seacat J. Nipple confusion: toward a formal definition. J Pediatr. 1995; 126 (Supplement): S125-S129.

30. Nakama L. Aleitamento materno e odontologia: atuação interdisciplinar e multiprofissional na construção da saúde bucal como direito de cidadania [tese]. Araçatuba: Faculdade de Odontologia da Universidade Estadual Paulista; 1999. 